\title{
Nociones populares sobre "dengue" y "rompehuesos", dos modelos de la enfermedad en Colombia'
}

\author{
Paulina Fajardo, ${ }^{2}$ Carlos Arturo Monje, ${ }^{2}$ Gladys Lozano, ${ }^{3}$ \\ Orlando Realpe ${ }^{4}$ y Luis Eduardo Hernández ${ }^{4}$
}

RESUMEN Objetivo. Hacer llegar a través de los niños mensajes educativos a las familias en Neiva, capital del Departamento del Huila, Colombia.

Métodos. Se desarrolló un proyecto educativo para incluir en el currículo de Ciencias $\mathrm{Na}$ turales y Educación Ambiental de los escolares de educación básica contenidos sobre el dengue, el vector y su control. Mediante encuestas y escalas tipo Likert se determinaron los conocimientos y las actitudes en los hogares de los escolares, y luego se complementaron con entrevistas abiertas que reflejaron aspectos culturales en el campo de la salud y la forma como esta enfermedad se integra a la vida de las personas. Antes y después de la intervención, se establecieron en los hogares los indices larvarios de infestación con el vector.

Resultados. Se determinó la presencia de un patrón cultural en torno a la enfermedad que supone una diferencia entre "rompehuesos" y "dengue". El "rompehuesos" corresponde al dengue clásico según el modelo popular de la enfermedad. Es aquella que puede ser afrontada con los recursos propios de la familia y la comunidad. La medicina institucional, por medio de sus organismos y agentes de salud, así como de los medios de comunicación, ha logrado superponer el otro modelo: el del dengue hemorrágico.

Discusión. Se examinan las alternativas de prevención adecuadas para las condiciones en que se presenta el ciclo del vector en Neiva, los modelos de percepciones acerca de la enfermedad presentes en la comunidad y los resultados de las encuestas, que orientaron la formulación $y$ desarrollo del programa educativo.

Palabras clave Dengue, dengue hemorrágico, Aedes aegypti.

La aparición del dengue y del dengue hemorrágico en América Latina siguió a la reinfestación por Aedes aegypti en la mayoría de los países de

\footnotetext{
Estudio realizado con el patrocinio de Colciencias mediante Convenio No. 112404-04-107-95-CT-26195.

2 Universidad Surcolombiana, Facultad de Ciencias de la Salud. Enviar correspondencia a Paulina Fajardo. Facultad de Salud. Calle 9, Carrera 14. Neiva, Huila, Colombia. Teléfono: (57-88) 718310; e-mail: dopafa@usurcolombia.com
}

donde este vector había sido erradicado (1). Se necesitaron 20 años para entender que las aplicaciones de insecticidas no tienen ningún impacto sobre la población del mosquito vector del dengue ni tampoco — según observan quienes han investigado los brotes epidémicos- un efecto significativo en la reducción de su transmisión (2).

En las medidas de control y prevención del dengue, la participación de la población urbana cumple un papel im- portante. Las grandes ciudades tropicales de las Américas se caracterizan por estar densamente pobladas y desarrollarse de manera caótica (3). La urbanización, rápida y desorganizada, sobrepasa la capacidad de los servicios sanitarios para atender a las necesidades de la población. Un ejemplo lo constituye el gran aumento de envases desechados que son hábitat favoritos de las larvas de A. aegypti y que proliferan sin ser recolectados. Pero aun 
cuando todos estos materiales inservibles fuesen eliminados adecuadamente, en los hogares seguirían utilizándose tanques, barriles y otros depósitos de agua para uso doméstico que son criaderos importantes de $A$. aegypti (4).

Cada vez se reconoce más que para que los programas de control de vectores den buenos resultados se necesita una amplia participación de la comunidad (5). De este modo se consigue no solo una mayor aceptación de las medidas de control, sino también mayor eficacia y continuidad (6). Sin embargo, para que tal participación tenga lugar, la comunidad involucrada debe compartir valores e intereses, además de estar física y psicológicamente unida por un sentido de pertenencia o identidad social. A propósito, Gordón (7) señala las dificultades que entrañó reunir a un grupo de trabajadores de la salud voluntarios en un barrio urbano de clase media baja de la República Dominicana. En Puerto Rico, Koss (8) encontró que un marco institucional tiene mayor poder de convocatoria; en este caso la institución agrupa a voluntarios para suministrar información o diseminar mensajes sobre el control de vectores.

Armada Gessa y Figueredo (1984) señalan que durante la epidemia de dengue hemorrágico en Cuba, casi todos los estamentos de la sociedad participaron en una campaña vertical de erradicación y control que incluyó actividades masivas de limpieza e inspecciones casa por casa (9). En Puerto Rico, República Dominicana y otros países de América Latina la participación voluntaria en medidas de control de A. aegypti fue promovida mediante campañas en escuelas, asociaciones comunitarias y medios masivos de comunicación. Si bien estas intervenciones aumentaron el conocimiento del público general acerca del dengue, los sitios de cría y el control del mosquito, no se lograron cambios significativos en la conducta de la gente. La razón de estos resultados limitados probablemente estribe en que no se entendió adecuadamente el mecanismo de la transmisión (10), en que hay preferen- cia por los enfoques curativos en vez de preventivos, o en que el dengue se confunde con otras enfermedades menos graves, como el resfriado común (11).

Se estima que en Colombia más de 15 millones de personas residen en zonas en riesgo de dengue hemorrágico (2). Según cifras proporcionadas por el Informe epidemiológico nacional, en 1997 se registraron 57985 casos de dengue (12). En 1990 el departamento del Huila, en el sureste del país, registró los primeros casos de dengue hemorrágico. En 1992 tuvo lugar una primera epidemia con 123 casos. Posteriormente se observó un ligero control de la enfermedad, sin que esta dejara de constituir un problema de salud pública. En 1997 se presentó una segunda epidemia de 330 casos, que se extendió a 1998 con 284 casos. Las poblaciones más afectadas fueron las de niños y adolescentes $(13,14)$. Estos brotes epidémicos fueron manejados mediante el control químico - fumigación para los mosquitos adultos, abatización para las larvas- y a través de mensajes educativos a la comunidad, con el fin de que ejecute acciones para el control de los criaderos del vector. Los resultados obtenidos con este tipo de intervención se consideran de eficacia limitada porque no conllevan cambios de comportamiento ni alternativas de participación. Por este motivo se consideró necesario buscar nuevas estrategias que comprometan a la comunidad a participar en la prevención y el control de la enfermedad.

\section{MATERIALES Y MÉTODOS}

En febrero de 1996 en Neiva, la capital del Huila, dos centros docentes lanzaron un proyecto que incluiría, dentro del currículo de ciencias naturales y educación ambiental, contenidos sobre el dengue, A. aegypti y su control. El propósito era llegar a los padres y familiares por medio de los niños, con mensajes educativos que estimularan una mejor respuesta a los programas contra el dengue que llevaban a cabo las autoridades de salud.
Otro componente del proyecto estaba dirigido a identificar la percepción que tiene el público sobre el dengue, con objeto de determinar si existía o no un modelo local sobre la enfermedad paralelo al modelo institucional. La población de estudio estuvo constituida por el total de hogares de los escolares de nivel básico (preescolar a $5^{\circ}$ grado) de los 18 establecimientos educativos de la comuna sur de Neiva. La muestra incluyó 520 hogares, distribuidos equitativamente entre los grupos de estudio y control. Los barrios de la comuna pertenecen a los estratos socioeconómicos bajo y medio bajo (15). El grado de cobertura en los servicios públicos básicos es alto. Las viviendas tienen paredes de ladrillo, pisos de cemento y techos tipo eternit. Solamente en $5,3 \%$ de las casas se vive en condiciones de hacinamiento (un solo cuarto para dormir). En la parte de atrás de la vivienda se encuentra el lavadero de ropa o alberca doméstica, techada solo en 33\% de los casos (16).

El estudio se realizó siguiendo a la población objetivo y la información se obtuvo mediante dos mediciones que se efectuaron al iniciar y al finalizar el estudio. Su ejecución comprendió cinco fases: 1) identificación de los conocimientos, actitudes y prácticas en los hogares, previo al programa educativo, 2) capacitación de los docentes de los centros de instrucción, 3) diseño y validación de los materiales educativos, 4) aplicación del programa educativo y 5) evaluación final, análisis e interpretación de resultados.

Para la recolección de la información sobre los conocimientos, actitudes y prácticas $(\mathrm{CAP})$ en la población se utilizó una combinación de técnicas cuantitativas y cualitativas. Las variables evaluadas comprendiron las causas del dengue, signos y síntomas (de dengue clásico y hemorrágico), cadena de transmisión, el vector y su hábitat, medidas de ordenamiento del medio para el control de A. aegypti, y participación de la comunidad en el control del vector.

Las técnicas cuantitativas incluyeron entrevistas estructuradas y mediciones escalares tipo Likert para las actitudes. Las técnicas cualitativas 
comprendieron la realización de entrevistas no estructuradas y la observación directa, procurándose entender la racionalidad de los comportamientos en los hogares y la comunidad para establecer las percepciones populares sobre el dengue. Las entrevistas no estructuradas fueron realizadas en hogares seleccionados al azar, tanto en el área de influencia del grupo de estudio como en la del grupo control. Dos miembros del grupo investigador se presentaron en cada uno de los hogares y, después de identificarse, solicitaron permiso para hacer algunas preguntas. El intercambio ocurrió como una conversación informal entre el habitante de la casa y uno de los investigadores, en tanto que el otro tomaba nota de las respuestas. Terminada la entrevista se escribía un resumen.

En los hogares se realizó además una encuesta entomológica que permitió establecer el índice de infestación de la vivienda y el índice de depósitos, antes y después de la aplicación de la estrategia educativa.

La información procedente de los instrumentos estandarizados se procesó electrónicamente mediante la utilización del programa Statistica para Windows y Excel. La información de carácter cualitativo se procesó manualmente atendiendo a criterios definidos por los investigadores, de acuerdo con los objetivos del estudio.

\section{RESULTADOS}

De acuerdo con la encuesta de conocimientos, las infecciones respiratorias y las fallas en la recolección de basura fueron consideradas por los jefes de hogar como los problemas sanitarios más importantes del barrio. En el cuadro 1 se presentan algunos conocimientos que indican el impacto logrado por el programa educativo. Si bien durante la encuesta postintervención se registró un aumento de los conocimientos del grupo de estudio con respecto a la encuesta previa a la intervención, no se pudo demostrar que las diferencias encontradas tuvieran significación estadística, ni se observaron diferencias significativas en los conocimientos del grupo de estudio y del grupo control.

En cuadro 2 se presentan las actitudes frente a las prácticas de prevención y control del vector que fueron valoradas como importantes e indicadoras del impacto logrado por el programa educativo. Después de la intervención, tanto el grupo de estudio como el grupo control mejoraron su actitud frente a las medidas para controlar el vector. La práctica que refleja una mejor actitud es la del lavado semanal de la alberca doméstica. El puntaje total obtenido en la escala de Likert muestra una actitud más favorable en el grupo de estudio, pero no se observa diferencia significativa entre este y el grupo control (cuadro 3).

A partir de los relatos obtenidos durante las entrevistas abiertas se realizó una categorización inductiva de los datos, se seleccionaron expresiones referidas a los temas de estudio y se trató de interpretar las percepciones de los actores sociales. En el cuadro 4 figuran las percepciones registradas en las entrevistas, de las cuales se deduce que "rompehuesos" y "dengue" representan dos modos de percibir la enfermedad viral transmitida por A. aegypti. El primero de ellos corresponde a la actitud tradicional y popular frente a la enfermedad. Por otra parte, lo que llaman "dengue" se asocia con el modelo

CUADRO 1. Conocimientos e indicadores del impacto del programa educativo en los grupos de estudio y control. Neiva, Huila, Colombia, febrero de 1996 y noviembre de 1998

\begin{tabular}{|c|c|c|c|c|}
\hline \multirow[b]{2}{*}{ Conocimiento } & \multicolumn{2}{|c|}{ Preintervención } & \multicolumn{2}{|c|}{ Postintervención } \\
\hline & $\begin{array}{c}\text { Estudio } \\
(\%)\end{array}$ & $\begin{array}{c}\text { Control } \\
(\%)\end{array}$ & $\begin{array}{c}\text { Estudio } \\
(\%)\end{array}$ & $\begin{array}{c}\text { Control } \\
(\%)\end{array}$ \\
\hline Nombre de la enfermedad: dengue & 14,2 & 16,7 & 37,8 & 39,5 \\
\hline La aspirina no es útil para curar el dengue & 10,8 & 5,6 & 19,2 & 12,9 \\
\hline Forma de transmisión: zancudo o mosquito & 29,8 & 29,3 & 52,3 & 52,9 \\
\hline Nombre del vector: Aedes aegypti & 2 & 1,4 & 26 & 11,8 \\
\hline Lugar donde ha visto las larvas: alberca & 2,7 & 3,3 & 14,5 & 11,4 \\
\hline Relaciona: recipientes con zancudos & 57,7 & 57,7 & 76,3 & 84,1 \\
\hline No cualquier zancudo transmite el dengue & 15,2 & 12,6 & 31,9 & 12,9 \\
\hline con el dengue & 20,7 & 21 & 40,9 & 31,2 \\
\hline Utilidad del abate: evita los "saltones" & 64,4 & 51,6 & 74,5 & 79,1 \\
\hline
\end{tabular}

CUADRO 2. Actitudes frente a las prácticas de prevención y control del vector indicadoras del impacto del programa educativo en los grupos de estudio y control. Neiva, Huila, Colombia, 1996

\begin{tabular}{llcllc}
\hline & \multicolumn{2}{c}{ Preintervención } & & \multicolumn{2}{c}{ Postintervención } \\
\cline { 2 - 3 } Conductas consideradas muy importantes & $\begin{array}{c}\text { Estudio } \\
(\%)\end{array}$ & $\begin{array}{c}\text { Testigo } \\
(\%)\end{array}$ & & $\begin{array}{c}\text { Estudio } \\
(\%)\end{array}$ & $\begin{array}{c}\text { Testigo } \\
(\%)\end{array}$ \\
\hline Lavar semanalmente la alberca & 46,4 & 39,5 & & 80,5 & 76,5 \\
$\begin{array}{l}\text { Tapar los recipientes donde se almacena agua } \\
\text { Guardar bajo techo los recipientes que }\end{array}$ & 39,0 & 33,5 & & 65,5 & 60,8 \\
$\quad$ almacenan agua & 36,3 & 25,6 & & 55,9 & 46,2 \\
$\begin{array}{l}\text { Colocar boca abajo los recipientes que } \\
\quad \text { almacenan agua }\end{array}$ & 36,3 & 34,9 & & 62,5 & 53,8 \\
Recoger objetos en desuso & 38,0 & 33,0 & & 64,4 & 55,4 \\
Limpiar los canales & 34,2 & 27,0 & & 52,1 & 49,2 \\
\hline
\end{tabular}


CUADRO 3. Puntaje total en la escala de actitudes de los hogares frente a las medidas de prevención y control del vector. Neiva, Huila, Colombia, febrero de 1996 y noviembre de 1998

\begin{tabular}{|c|c|c|c|c|c|c|c|c|}
\hline \multirow[b]{3}{*}{ Puntaje } & \multicolumn{4}{|c|}{ Grupo de estudio } & \multicolumn{4}{|c|}{ Grupo testigo } \\
\hline & \multicolumn{2}{|c|}{ Pretest } & \multicolumn{2}{|c|}{ Postest } & \multicolumn{2}{|c|}{ Pretest } & \multicolumn{2}{|c|}{ Postest } \\
\hline & No. & $\%$ & No. & $\%$ & No. & $\%$ & No. & $\%$ \\
\hline Muy desfavorable $(<$ de 12$)$ & 4 & 1,4 & 1 & 0,4 & 0 & 0.0 & 0 & 0.0 \\
\hline Desfavorable (12 a 24) & 3 & 1,0 & 2 & 0,8 & 7 & 3,3 & 2 & 0,8 \\
\hline Indeciso (25 a 36) & 188 & 63,5 & 87 & 33,4 & 146 & 67,9 & 81 & 30,8 \\
\hline Favorable (37 a 48) & 101 & 34,1 & 156 & 60 & 61 & 28,4 & 178 & 67,6 \\
\hline Muy favorable (49 a 60) & 0 & 0,0 & 13 & 5,0 & 1 & 0,4 & 2 & 0,8 \\
\hline Sin respuesta & 0 & 0,0 & 1 & 0,4 & 0 & 0.0 & 0 & 0,0 \\
\hline Total & 296 & 100 & 260 & 100 & 215 & 100 & 263 & 100 \\
\hline
\end{tabular}

institucional, difundido a través de los medios de comunicación y de los agentes de salud.

En julio de 1996, durante la encuesta preintervención de los índices de infestación con larvas de $A$. aegypti, que la comunidad denomina "saltones", se encontró que tanto el índice de casas como el índice de criaderos eran semejantes en ambos grupos. Dada su presencia en todos los hogares, el criadero más importante fue el tanque para el lavado de ropa o alberca doméstica. En los patios se encontró una gran cantidad de depósitos diversos que constituyen criaderos potenciales para el vector. En la encuesta postintervención, efectuada en octubre de 1998, se halló que en ambos grupos los índices de infestación, tanto de las casas como de los criaderos, fueron superiores a los determinados por la encuesta preintervención (cuadro 5).

Con objeto de determinar cuáles eran los "recipientes diversos" encontrados, en agosto de 1996 se realizó una encuesta entomológica en 43 viviendas de la comuna. En total se inspeccionaron 1183 recipientes. Setenta y un por ciento de ellos eran botellas, de las cuales solo 5\% contenían agua y ninguna mostró la presencia de larvas de $A$. aegypti. Los platos y vasijas utilizados como comederos y bebederos de animales fueron los depósitos que más a menudo contenían agua (25\%). Aunque solo alcanzó $0,4 \%$, el mayor índice de infestación tuvo lugar en los criaderos de animales (cuadro 6). Cuando se preguntó a los residentes qué uso daban a los recipientes diversos encontrados en los patios de las viviendas, se halló que $75 \%$ eran objetos considerados reciclables que la familia esperaba vender como una fuente de ingresos $y$ $6,4 \%$ eran bebederos y comederos de animales domésticos que por lo general se lavan diariamente. Solo $10 \%$ de los objetos se consideraban inservibles y eliminables en la basura.

Dentro del programa educativo se incluyen un curso dirigido a los docentes para capacitarlos acerca del dengue, el vector y su control; materiales didácticos elaborados para facilitar el aprendizaje y apoyar la ejecución de las programaciones curriculares, y un Servicio de Vigilancia Infantil contra el Dengue (SEVID), proyecto que desarrollan los escolares del quinto grado de educación básica en la comunidad que rodea a la escuela con miras a adoptar prácticas de saneamiento ambiental que contribuyan a mejorar su calidad de vida y a estimular en el grupo familiar una mejor respuesta al problema del dengue. El SEVID es una estrategia pedagógica eficaz porque, además de gozar de aceptación por parte de los padres de familia, ha contribuido a fortalecer la preparación de los escolares y ha mejorado notablemente las relaciones de la escuela con la comunidad.

Las observaciones y alternativas de prevención y control, adecuadas a las condiciones en que se presenta el ciclo de vida del vector en Neiva y a los modelos de percepción de las enferme- dades presentes en la comunidad, así como los resultados de las encuestas entomológicas y de CAP, orientaron la formulación y el desarrollo del programa. Con este propósito estos materiales fueron colocados al alcance de los profesores de los centros docentes para ser incorporados a los materiales educativos que ellos diseñaban y se incluyeron además en el libro de texto Educación para la salud con énfasis en la prevención y control del dengue, que orienta el programa educativo y constituye una guía para los maestros que participan.

\section{DISCUSIÓN}

Según datos de la Secretaría de Salud municipal, los índices de infestación larvaria en las viviendas de la comuna sur durante los años del estudio fueron de $24,9 \%$ en 1996 y de 21,1\% en 1997 y 1998 (17). En los hogares de los escolares los índices encontrados antes de la aplicación de la estrategia educativa fueron más bajos que los notificados para 1996, probablemente porque fueron medidos en julio, cuando el nivel de las lluvias llegó solamente a 5,1 mm. Por el contrario, en la encuesta postintervención los índices de infestación en los hogares fueron medidos en octubre, durante el período de lluvias $(260,7 \mathrm{~mm})$ (18), cuando los recipientes diversos que constituyen los "criaderos potenciales" adquieren peligrosidad. Esto puede explicar los mayores índices de infestación notificados para 1998. En el grupo estudiado se observó, además, que durante el período del estudio se instalaron en el sector varios negocios de reparación de neumáticos que alcanzaron un índice de positividad cercano al 19\% (cuadro 5). En el grupo testigo, debido a las fallas en el suministro de agua, se presenta un mayor número de tanques elevados que no se tapan adecuadamente. No fue posible establecer ninguna diferencia estadísticamente significativa entre los índices de los dos grupos.

El aumento del número de casos de dengue hemorrágico durante 1997 y el primer semestre de 1998 se enmarca en 
CUADRO 4. Conocimiento de la enfermedad según su identificación como "dengue" o "rompehuesos". Neiva, Huila, Colombia, 1996

\begin{tabular}{|c|c|c|}
\hline & "Rompehuesos" & "Dengue" \\
\hline Síntomas & $\begin{array}{l}\text { - Es una fiebre alta con fuerte dolor en los huesos, } \\
\text { malestar general y decaimiento. } \\
\text { - Fiebre interna con desaliento y escalofríos. } \\
\text { - Es como una gripe fuerte, sin tos y con mucho dolor en el } \\
\text { cuerpo que lo lleva a uno a la cama. } \\
\text { - No dan ganas de comer y produce mucha sed. }\end{array}$ & $\begin{array}{l}\text { - Es otra clase de gripe, más brava que la "rompehuesos", } \\
\text { da con hemorragia y vómito. } \\
\text { - Debilita más a la persona y puede causar la muerte. } \\
\text { - Es similar a la "rompehuesos", da con una fiebre muy } \\
\text { alta. } \\
\text { - Se presenta con brote en el cuerpo. }\end{array}$ \\
\hline Causas & $\begin{array}{l}\text { - Aparece repentinamente, no se puede evitar, nunca } \\
\text { avisa. } \\
\text { - No es transmisible como la gripe. } \\
\text { - Se presenta en forma de epidemia, es como una peste. } \\
\text { - Es un virus volante que se produce por guardar agua } \\
\text { sucia y no lavar los tanques de agua. } \\
\text { - Al beber el agua con "saltones" se puede producir la } \\
\text { "rompehuesos". }\end{array}$ & $\begin{array}{l}\text { - Se produce por zancudos muy infectados provenientes de } \\
\text { aguas contaminadas. } \\
\text { - Proviene de mosquitos que se crían en albercas y } \\
\text { recipientes con agua. } \\
\text { - Es un zancudo pequeño que sale de las aguas podridas y } \\
\text { pone los huevos dentro de la sangre cuando pica. } \\
\text { - Es un zancudo que inyecta lo malo y quita lo bueno. } \\
\text { - Es un virus que transportan los zancudos. } \\
\text { - Da porque en los patios se crían animales, como cerdos y } \\
\text { gallinas. }\end{array}$ \\
\hline Curación & $\begin{array}{l}\text { - Automedicación con medicamentos y con productos } \\
\text { naturales. } \\
\text { - El "matrimonio": mezcla de antibiótico, analgésico y } \\
\text { antipirético (tetraciclina, aspirina y dristán). } \\
\text { - Infusiones o emplastos de zábila, matarratón o pelá. } \\
\text { - Beber líquido en abundancia. }\end{array}$ & $\begin{array}{l}\text { - Tratamiento médico. } \\
\text { - Tomar abundante líquido. }\end{array}$ \\
\hline El vector y su control & $\begin{array}{l}\text { - Prácticamente todos han visto al vector el "saltón". } \\
\text { - Se desconoce su relación con el zancudo y la } \\
\text { enfermedad. } \\
\text { - Pocos creen que del "saltón" sale el zancudo. } \\
\text { - El "saltón" se origina en las albercas cuando permanecen } \\
\text { largo tiempo sin lavar, están expuestas al polvo, cogen } \\
\text { lama o sedimento. } \\
\text { - Se da en las aguas de lluvia, en chuquios o charcos. }\end{array}$ & $\begin{array}{l}\text { - Identificación generalizada de la larva (el saltón), } \\
\text { principalmente en la alberca doméstica. } \\
\text { - No se asocia al vector (el saltón) con la aparición del } \\
\text { zancudo. } \\
\text { - El zancudo se asocia con la presencia de basuras y } \\
\text { suciedad. } \\
\text { - Algunas personas consideran que el zancudo proviene } \\
\text { del "saltón". } \\
\text { - Atribuyen el dengue a un zancudo no determinado. } \\
\text { - El "saltón" no es ofensivo, pero el zancudo sí lo es. } \\
\text { - Se reconoce como principal medida de control la } \\
\text { - utilización del abate y la fumigación. } \\
\text { - Ha escuchado que el abate sirve para descontaminar el } \\
\text { agua y matar los mosquitos. } \\
\text { - Casi todas las personas han visto el carro de salud } \\
\text { pública fumigando en las calles. }\end{array}$ \\
\hline
\end{tabular}

el brote de esta enfermedad que se registró en todo el país y que afectó especialmente a las ciudades de Bucaramanga, Cali y Neiva. Tal incremento fortaleció la vigilancia epidemiológica, llevando a la búsqueda de casos potenciales en las instituciones de salud, lo que explica el gran número de casos diagnosticados. Como factor de riesgo para estas dos epidemias se observó la presencia del Fenómeno del Pacífico o de "El Niño", período en el cual se registraron cambios en el medio ambiente, como sequías y aumento de la temperatura, que favorecen el incremento de la población del vector al disminuir la duración de su fase acuática.
Los conocimientos sobre el dengue identificados en los hogares podrían provenir de los mensajes educativos difundidos por los medios de comunicación, dado que la enfermedad es endémica en el área y que ya en 1992 se había presentado un brote de dengue hemorrágico. Las fumigaciones realizadas durante los brotes presentados en 1997 y 1998 y las visitas periódicas de los funcionarios de salud para aplicar el abate en los lavaderos de ropa, así como las campañas de prevención difundidas por los medios de comunicación, de alguna manera han contribuido a mejorar los conocimientos de las poblaciones de estudio y control y por esto no se obser- van diferencias en los resultados posteriores a la evaluación.

Es importante señalar que el grupo de estudio obtuvo puntajes más altos en conocimientos específicos presentados dentro del programa educativo, tales como "la aspirina no es útil para curar el dengue", el nombre del vector (Aedes aegypti) y "no cualquier zancudo transmite el dengue" (cuadro 1), conocimientos que no se incluyen en los mensajes difundidos por las autoridades de salud. En cuanto a las actitudes, el grupo de estudio recibió mejor puntaje en el "lavado semanal de la alberca doméstica", una práctica que fue subrayada en los mensajes difundidos 
CUADRO 5. Índice de infestación por larvas de Aedes aegypti pre y postintervención en los hogares de los escolares del estudio. Neiva, Huila, Colombia, julio de 1996 y octubre de 1998

\begin{tabular}{|c|c|c|c|c|c|c|c|c|}
\hline \multirow[b]{3}{*}{ Encuesta } & \multicolumn{4}{|c|}{ Preintervención } & \multicolumn{4}{|c|}{ Posintervención } \\
\hline & \multicolumn{2}{|c|}{ No. } & \multicolumn{2}{|c|}{$\%$ positividad } & \multicolumn{2}{|c|}{ No. } & \multicolumn{2}{|c|}{$\%$ positividad } \\
\hline & Estud. & Control & Estud. & Control & Estud. & Control & Estud. & Control \\
\hline CASAS: & 351 & 407 & 10,3 & 12,5 & 264 & 299 & 24,6 & 32,1 \\
\hline CRIADEROS: & 2828 & 3283 & 1,4 & 1,5 & 2085 & 2339 & 3,4 & 4,4 \\
\hline Albercas & 392 & 458 & 8,9 & 11,1 & 334 & 413 & 22,8 & 22,2 \\
\hline Tanques & 199 & 354 & 0,0 & 0,0 & 162 & 292 & 0,0 & 3,7 \\
\hline Neumáticos & 3 & 7 & 100,0 & 0,0 & 37 & 31 & 18,9 & 0,0 \\
\hline Árboles & 1 & 0,0 & 0,0 & 0,0 & 3 & 0,0 & 0,0 & 0,0 \\
\hline Diversos & 2233 & 2464 & 0,1 & 0,0 & 1549 & 1603 & 0,1 & 0,0 \\
\hline
\end{tabular}

CUADRO 6. Índice de infestación por larvas de Aedes aegyptien 1183 criaderos diversos. Neiva, Huila, Colombia, agosto de 1996

\begin{tabular}{lccc}
\hline \multicolumn{1}{c}{ Recipiente } & $\begin{array}{c}\text { Inspeccionados } \\
\%\end{array}$ & $\begin{array}{c}\text { Positivo } \\
\%\end{array}$ & $\begin{array}{c}\text { Con agua } \\
\%\end{array}$ \\
\hline Tarros y galones & 6,5 & 2,5 & 5,0 \\
Latas & 2,0 & 0,0 & 0,0 \\
Botellas & 70,8 & 0,0 & 5,0 \\
Frascos y floreros & 6,8 & 1,2 & 12,5 \\
Platones, tinas y baldes & 3,9 & 2,1 & 7,5 \\
Vasijas y platos plásticos & 5,1 & 3,2 & 25,0 \\
Vasijas metálicas, ollas & 3,3 & 5,1 & 20,0 \\
Tubos de plástico & 0,4 & 0,0 & 12,5 \\
Bacinillas & 0,8 & 0,0 & 0,0 \\
Criaderos de animales & 0,4 & 40,0 & 12,5 \\
Total & 100 & 0,8 & 100 \\
\hline
\end{tabular}

por el programa y que busca modificar en la comunidad la percepción del larvicida abate como única medida de control (cuadro 2).

Los conocimientos populares difieren de los formales o institucionales en la percepción de la enfermedad, su forma de transmisión y su curación y control. La "rompehuesos", de acuerdo con los síntomas expresados, se concibe como una enfermedad caracterizada por fiebre alta, dolor en los huesos y el cuerpo, malestar general, decaimiento, pérdida del apetito y mucha sed. Se asocia con una gripe fuerte que produce fiebre, desaliento y escalofríos que incapacitan a la persona. Produce, al decir de la gente, "una fiebre que le duele a uno todo el cuerpo como si le hubieran pegado". En cambio, el "dengue" se considera otra clase de gripe, más fuerte que la "rompehuesos", en algunos casos con hemorragia y vómito. $\mathrm{Da}$ con fiebre muy alta y un brote en el cuerpo, provoca mayor debilitamiento y puede producir la muerte. Según lo explican las personas entrevistadas, "es otra clase de gripe, da con hemorragia y vómito, es más brava que la rompehuesos, debilita más a la persona y si no le pone cuidado se va al otro charco". La diferencia fundamental se relaciona con la intensidad de los síntomas y manifestaciones de la enfermedad, que para el caso del dengue pueden adquirir carácter letal.

Con relación a las causas, la "rompehuesos" aparece repentinamente, no es transmisible y se presenta en forma de epidemia. "Ataca a todo el mundo porque viene de la atmósfera contami- nada". El "dengue", en cambio, es transmitido por zancudos muy infectados provenientes de aguas contaminadas, mosquitos que se crían en albercas y en recipientes con agua. Según la percepción popular entonces, la diferencia radica en que la causa de la "rompehuesos" es desconocida, mientras que la transmisión del "den-

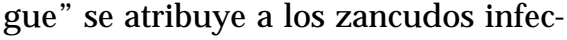
tados porque se crían en aguas sucias.

El tratamiento para la cura de "rompehuesos" consiste en una combinación de medicamentos, popularmente denominada "matrimonio" (antibiótico, analgésico y antipirético), recomendados por un ayudante de farmacia, un familiar o amigo. También se utilizan productos naturales y se aconseja beber líquidos en abundancia: "Tomar agua de matarratón que le quita la fiebre y le saca todo eso por que lo pone a uno a vomitar". En el caso del "dengue", también hay que tomar gran cantidad de líquidos, pero se necesita un tratamiento médico. Se puede concluir que la "rompehuesos" es una enfermedad poco peligrosa, usualmente curada en la casa, mientras que el "dengue" necesita atención médica.

En cuanto al vector y su control, prácticamente todos los entrevistados dijeron haber visto las larvas (los saltones) en las albercas cuando permanecen largo tiempo sin lavar, pero pocos asocian al saltón con la aparición del zancudo o conocen su relación con la enfermedad "rompehuesos": "No sé si los saltones tienen relación con la rom- 
pehuesos, pero me han dicho que sí con el dengue hemorrágico". Se sabe que el "dengue" es transmitido por el zancudo. El saltón es inofensivo pero el zancudo no lo es y, además, se lo asocia con la presencia de aguas sucias. Es decir, que la gente conoce la forma larvaria del vector con el nombre de "saltón", pero no lo asocia con la presencia de zancudos ni con la enfermedad "rompehuesos".

Casi todos los encuestados conocen la bolsita con abate y consideran que sirve para descontaminar el agua y matar a los saltones. También han visto el carro de salud pública que hace la fumigación en las calles para eliminar los zancudos que transmiten el dengue.

En la población estudiada, la diferenciación entre la "rompehuesos" y el "dengue" guarda relación con la coexistencia de las tradiciones médicas popular e institucional. Ante la presencia del dengue, la comunidad ha desarrollado sus propias formas de comprender, interpretar y tratar la enfermedad. Tales formas abarcan desde el reconocimiento de los síntomas y manifestaciones, pasando por la atribución de factores de causalidad, hasta las medidas de prevención y curación. El dengue clásico corresponde, según el modelo popular de la enfermedad, a "rompehuesos", que puede ser afrontada con los recursos propios de la familia y la comunidad. La medicina institucional, mediante sus organismos y agentes de salud, así como los medios de comunicación, ha logrado superponer el otro modelo de la enfermedad: el dengue en su forma hemorrágica. Para la comunidad el "dengue" es, entonces, la entidad patológica que reviste mayor gravedad y por lo tanto requiere la atención médica institucional. La presencia del zancudo como agente transmisor se atribuye a las malas condiciones sanitarias. En consecuencia, la responsabilidad del control del vector se delega a las instituciones de salud del Estado.

La presencia de los dos modelos de la enfermedad interfiere con los mensajes educativos que las autoridades de salud diseminan por los medios de comunicación, ya que la comunidad no se considera afectada por el "dengue" y, en consecuencia, no participa en los esfuerzos de prevención y control que se le solicitan.

Tradicionalmente los educadores de la salud piensan que solo ellos conocen la respuesta correcta, sin percatarse de que los miembros de la comunidad tienen sus propias ideas y percepciones sobre los problemas de salud (19). La experiencia demuestra que, para desarrollar y ejecutar programas de control del dengue basados en la participación comunitaria, hace falta reunir datos sociales o culturales que permitan entender mejor las percepciones y prácticas de los miembros de la comunidad en relación con la enfermedad. Asimismo, para que el mensaje llegue a la comunidad, cualquier programa educativo que se implemente debe tener en cuenta los dos modelos.

Un modo de estimular la participación de la comunidad consiste en descentralizar el diseño y la producción de materiales educativos, de tal suerte que puedan tomarse en consideración las características ecológicas, sociales y culturales de cada comunidad, en particular donde se va a ejecutar el programa. De esta forma se ganará en cuanto a efectividad e impacto sobre la población, dado que los contenidos de los programas estarán mejor adaptados a las condiciones, conceptos, percepciones y terminología locales (20). Sin embargo, no hay que olvidar que los mensajes apropiados para la salud no siempre se traducen en un cambio de conducta (21). La comunicación en sí no necesariamente tiene un efecto en los índices larvales de $A$. aegypti, sino que más bien debe entenderse como parte de un amplio programa para el control de las enfermedades transmitidas por vectores.

Aun cuando el impacto del programa solo podrá ser evaluado a largo plazo, sus resultados en términos educativos seguramente radican en la continuidad que le confiere su inclusión en el currículo, formando parte de los contenidos que los escolares desarrollan a lo largo de todo el ciclo de seis años. Es fundamental, además, que su seguimiento esté en manos de autoridades comprometidas con la formación de nuevas generaciones mejor educadas en salud. En el corto plazo, en cambio, sí es posible reducir las tasas de infestación larvaria del vector, pero siempre y cuando se diseñen e implementen programas que cuenten con la participación de agrupaciones comunitarias que contribuyan a diseminar los mensajes educativos.

\section{REFERENCIAS}

1. Organización Panamericana de la Salud. Dengue y dengue hemorrágico en las Américas: guías para su prevención y control. Edición de 1995. Washington, D.C.: OPS; 1995. (Publicación científica 548).

2. Gubler DJ. Aedes aegypti and Aedes aegyptiborne disease control in the 1990s: top down or bottom up. Am J Trop Med Hyg 1989;40(6): 571-578.

3. Koss J, Roman F. Current technologies for vector control and prevention of vector-borne diseases. Symposium "Community participation and control efforts in developing countries", Annual Meeting of the American Mosquito Control Association, New Orleans, 1991.

4. Chow CY, Gratz NG, Tonn RJ, Self LS, Pant YC. Control de las epidemias transmitidas por Aedes aegypti. 1978. (Documento inédito WHO/VBC/77.660).

5. Gubler DJ, Clark GG. Community involvement in the control of Aedes aegypti. Acta Tropica 1996;61(2):169-179.

6. Winch P, Kendall C, Gubler D. Community participation in the control of vector-borne di- sease. A review of selected activities in Latin America. Symposium "Community participation and control efforts in developing countries", Annual Meeting of the American Mosquito Control Association, New Orleans, 1991.

7. Gordon AJ. Mixed strategies in health education and community participation: an evaluation of dengue control in the Dominican Republic. Health and Education Research 1988; 3(4):399-419.

8. Koss J. Final report: Innovative educational approaches to the prevention of dengue and 
dengue hemorrhagic fever in Puerto Rico. Albuquerque: Department of Psychiatry, University of New Mexico; 1988.

9. Armada Gessa JA, Figueredo R. Ordenamiento del medio en el programa de erradicación de Aedes aegypti. Cuba, 1984. Bol Oficina Sanit Panam 1987;102:(3)237-244.

10. Winch P, Kendall C, Leontsini E. Community participation in Aedes aegypti control programmes. En: Uren MF, Blok J, Manderson LH, eds. Arbovirus Research in Australia: Proceedings Fifth Symposium, August 28, September 1, 1989, Brisbane, Australia. Brisbane: Commonwealth Scientific and Industrial Research Organisation; 1990. Pp. 224-228.

11. Gordon AJ, Rojas Z, Tidwell M. Cultural factors in Aedes aegypti and dengue control in Latin America: a case study from the Dominican Republic. Int Q Commun Health Educ 1990;10:193-121.

12. Informe Epidemiológico Nacional. Evaluación epidemiológica del dengue hemorrágico en Colombia. Santa Fe de Bogotá. Minsalud; INS 1997;2(19).
13. Informe Epidemiológico Nacional. Sistema Alerta Acción 1998;3(9).

14. Secretaría de Salud Departamental. Diagnóstico de Salud, Departamento del Huila, Colombia; 1996-1998.

15. Idrobo JC. Informe final del proyecto de revisión de la estratificación socioeconómica de Neiva. Neiva, Huila, Colombia: Oficina de Planeación, Municipio de Neiva. Febrero de 1999. P. 20.

16. Benavides M, Realpe O. Evaluación del tiempo en que Aedes aegypti coloniza y desarrolla su fase acuática en los lavaderos de ropa de los barrios sur-orientales de Neiva. Biomédica 1997;14(4):282-285.

17. Secretaría de Salud Municipal de Neiva, Huila, Colombia. Informe de actividades de control, enfermedades transmitidas por vectores, 1996-1998. Neiva, Huila, Colombia: Secretaría de Salud Municipal de Neiva.

18. Instituto de Hidrológica, Meteorología y Estudios Ambientales. Registros meteorológicos para el departamento de Huila. Neiva, Huila, Colombia: IDEAM; 1996.
19. Leontsini E, Helitzer D. Communication approaches in community-based mosquitoborne disease control programs. Symposium "Community participation and control efforts in developing countries". Presentado en la reunión anual de la American Mosquito Control Association, New Orleans, 1991.

20. Lloyd L, Winch P, Ortega J, Kendall C. The design of a community-based health education intervention for the control of Aedes aegypti. Am J Trop Med Hyg 1994; 50(4):401-41.

21. Gramiccia G. Health education in malaria control. Why has it failed? World Health Forum 1981;2(3):385-393.

Manuscrito recibido el 26 de octubre de 2000 y aceptado

ABSTRACT Objective. To deliver, through children, educational messages to families in Neiva, the capital city of the department of Huila, in Colombia.

\section{Popular notions surrounding "dengue" and rompehuesos, two models of the disease in Colombia}

Methods. An educational project was developed to include, in schoolchildren's natural sciences and environmental education curriculum, basic education on dengue, its vector, and its control. Through surveys and Likert scales, the knowledge and attitudes in the schoolchildren's homes were determined. This was complemented with open-ended interviews that reflected cultural aspects in the area of health and how dengue disease is a part of people's lives. Before and after the intervention, larval infestation indices were determined for the schoolchildren's homes.

Results. A cultural pattern was found for the disease that differentiated between rompehuesos (breakbone fever) and "dengue". Rompehuesos corresponds, in popular terms, to classical dengue, a disease that can be dealt with using family and community resources. Institutional medicine, by means of its health agencies and health workers, as well as the communications media, has managed to superimpose another model: that which is associated with dengue hemorrhagic fever.

Discussion. Alternatives are considered for prevention efforts that would be sufficient for the conditions in which the vector's cycle occurs in Neiva. Also presented are the community's disease model and the survey results. Together, these elements can guide the formulation and development of educational efforts. 
para publicación, tras revisión, el 27 de junio de 2001. 